\title{
Enhanced hydrolysis of organic contaminants by metal oxide nanoparticles: role of crystallinity and exposed facets
}

\author{
WEI CHEN ${ }^{1}$, CHUANJIA JIANG ${ }^{1}$
}

${ }^{1}$ College of Environmental Science and Enginering, Nankai University, 38 Tongyan Rd., Tianjin 300350, China; chenwei@nankai.edu.cn

Naturally occurring metal oxide nanoparticles are ubiquitous in the environment; with the rapidly increasing production and use of engineered nanomaterials, the incidental and accidental environmental release of metal oxide nanomaterials is inevitable. Understanding the interactions between metal oxide nanoparticles with environmental pollutants is critical for the assessment of nano implications. Additionally, development of nanotechnologybased applications for contaminant removal also calls for improved understanding of the structure-activity relationship controlling the nanoparticles-contaminant interactions. Here, we reveal the critical roles of crystallinity and exposed facets of metal oxide nanoparticles in determining their catalytic efficiencies for the hydrolysis reactions of organic contaminants. We show that crystallinity and exposed facets of metal oxide nanocrystals (e.g., $n \mathrm{TiO}_{2}, n \mathrm{Fe}_{2} \mathrm{O}_{3}$, and $n \mathrm{FeOOH})$ can significantly affect the efficiencies of the nanomaterials in catalyzing environmentally relevant acidand base-catalyzed hydrolysis reactions, by controlling both adsorption affinities and concentrations of surface reactive moieties of nanocrystals. Crystalline phase and exposed facets affect adsorption affinities of metal oxide nanocrystals by modulating surface hydrophobicity of the nanocrystals and controlling the abilities of metal atoms to form complexes with contaminants. Crystalline phase and exposed facets also determine the activity of surface catalytic sites on metal oxide nanocrystals by dictating the concentration and strength of surface unsaturated metal atoms, which along with the deprotonated hydroxyl groups attached can serve as acidic or basic sites to catalyze both acid- and base-promoted hydrolysis. 